\title{
NUMERICAL INVESTIGATIONS OF AERODYNAMIC PROPERTIES OF A PROPELLER BLOWN CIRCULATION CONTROL SYSTEM ON A HIGH WING AIRCRAFT
}

\author{
$50^{\text {th }} 3$ 3F INTERNATIONAL CONFERENCE ON APPLIED AERODYNAMICS \\ Toulouse, France, 29-30 March - 01 April 2015 \\ Dennis Keller ${ }^{(1)}$, Ralf Rudnik ${ }^{(1)}$ \\ (1) Insitute of Aerodynamics and Flow Technology, German Aerospace Center, Lilienthalplatz 7, 38108
Braunschweig, Germany, Email: dennis.keller@dlr.de, ralf.rudnik@dlr.de
}

The contribution gives an overview over a wide range of CFD simulations, which were performed in the course of the German collaborative research center $\mathbf{8 8 0}$ in order to investigate the aerodynamic properties of a complete turboprop powered transport aircraft in landing configuration with a circulation control high-lift system. The main purpose of the contribution is to highlight aerodynamic and flight mechanical aspects of the integration of lift augmentation technologies into the design of a short take-off and landing aircraft concept. In this context, the influence of engine nacelles and thrust on the stall behavior and the following improvements due to the use of a nacelle strake are discussed. Furthermore, static longitudinal and lateral stability as well as the dynamic longitudinal stability are investigated. While circulation control itself has a rather small impact on the stability, the impact of engine thrust in conjunction with circulation control is considerable. Additionally, the one engine inoperative case was simulated. For some flow and engine conditions, the resulting yawing moments are more than twice as high as the actual yawing moments due to asymmetric thrust.

\section{INTRODUCTION}

A novel transport aircraft design with short take-off and landing (STOL) capabilities is investigated within the German collaborative research center 880 (CRC 880). To achieve STOL capabilities, the aircraft's high-lift system utilizes circulation control (CC) in conjunction with a smart droop nose and wing-mounted turboprop engines to allow the requested lift augmentation.

The potential of lift augmentation due to propeller slipstream and especially circulation control is already well known [1], [2], [3], [4]. However, this potential can be significantly deteriorated by aerodynamic installation effects resulting from the integration of these technologies into the aircraft. Therefore, the estimation of these integration effects and thus the evaluation of the high-lift performance of a state-of-the-art aircraft configuration equipped with propeller and circulation control is one of the main tasks of the current investigation.

Furthermore, the use of these technologies can have a critical influence on the flight mechanical properties of the aircraft. Obviously, low take-off and landing speeds lead to small aerodynamic forces on the control surfaces. Additionally, the lift augmentation systems influence the flow conditions downstream of the wing in the region of the tail plane. Investigations of similar aircraft configurations suggest that the aircraft's stability can be negatively affected [5], even though the impact is rarely documented [6], [7], [8]. In particular, detailed information about the origin of the change in the stability behavior is lacking due to the experimental character of these investigations. Therefore, the impact of the lift augmentation systems on the aircraft's stability behavior and its origin is the second question, which shall be addressed. In order to investigate the impact of these technologies in the present context, a comprehensive aerodynamic database had to be created. The focus is laid on the aerodynamic performance characteristics, but also on flight dynamics aspects of the whole aircraft, such as the determination of static and dynamic derivatives. The basic aircraft configuration itself has been defined as part of CRC 880 [9]. 
RANS simulations of the trimmed aircraft in landing configuration with thrust and circulation control were performed in order to investigate longitudinal and lateral static stability [10].

For the study of the dynamic behavior, two dimensional as well as three dimensional URANS calculations were carried out.

Additionally, tail-off calculations led to important findings regarding the sensitivity of the flowfield at the location of the horizontal stabilizer (HTP).

As asymmetric thrust of wing mounted propeller aircraft can result in significant forces and moments, especially on a highly loaded wing [11] [12], the case of one engine inoperative (OEI) was also simulated.

In addition to the analysis of the aircraft behavior, the simulations also aimed at further improving the preliminary aircraft design. As a result, revised tail planes were integrated into the design. Furthermore, also the high-lift performance could be further improved by the use of a nacelle strake.

The paper gives an overview of the wide range of highly complex numerical simulations, which were performed in this context.

Selected results of the investigations are presented and analyzed.

\section{NUMERICAL METHOD}

The calculations are performed with the DLR TAU code [13], which is based on an unstructured finite volume approach for solving the Reynoldsaveraged Navier-Stokes equations. For the present investigation, the implicit LUSGS scheme is used for time stepping and a central scheme with scalar dissipation for the spatial discretization of the convective fluxes. Turbulence effects are modelled with the original Spalart-Allmaras formulation (SA) [14] with modifications to account for vortical and rotational effects based on the Spalart-Shur correction [15]. In order to model the turboprop engines, actuator disks based on the 2D blade element theory are implemented. In this way, the local load of the propellers is calculated based on a given radial distribution of force coefficients along the blades and the local flow conditions [16]. Since the actuator disk model itself is nontransient and does not consider the movement of single propeller blades, the model is inappropriate when unsteady phenomena such as blade wake and vortex shedding are of interest. However, the use of an actuator disk model can greatly reduce computational costs due to the minimization of the mesh size and the use of steady simulations, while main aspects of the propeller impact such as an inhomogeneous change of the flow momentum and swirl are considered. The procedure already showed robust behavior and good results in various applications such as the simulation of a contra-rotating open rotor [17] and a circulation controlled high-lift wing-propeller configuration, which is similar to the underlying configuration [18].

\subsection{Trim procedure}

In order to analyze the longitudinal and lateral stability behavior, the aircraft was trimmed. Besides balancing the pitching moment coefficient $\left(c_{\mathrm{My}}\right)$, the trim procedure levels out thrust and drag (drag coefficient: $c_{D}$ ) in case of simulations with active engines and optionally achieves a targeted lift coefficient $\left(c_{\mathrm{L}}\right)$. The trim procedure therefore solves for the HTP's trim angle ( $\eta$ ) and optionally for the propeller blade pitch angle $(\beta)$ and the angle of attack ( $\alpha$ ) with a fully automated newton method (eq. 1). In case of thrust trimming, the target drag is either given by the user or calculated for a balanced flight at a given glide slope based on the previous newton iteration (eq. 2). The necessary initial Jacobi matrix is evaluated by linear differences. Alternatively, it can be manually predefined. In the course of the iterative trim procedure it is then adapted based on the results of the latest iterations. The adjustment of the HTP trim angle calls for a modification of the geometry and the CFD mesh, which is realized by deformation based on radial basis functions [19].

$$
\begin{gathered}
{\left[\begin{array}{ccc}
c_{M y \eta}^{i-1} & c_{M y \beta}^{i-1} & c_{M y \alpha}^{i-1} \\
c_{D \eta}^{i-1} & c_{D \beta}^{i-1} & c_{M D \alpha}^{i-1} \\
c_{L \eta}^{i-1} & c_{L \beta}^{i-1} & c_{L \alpha}^{i-1}
\end{array}\right]\left[\begin{array}{l}
\Delta \eta \\
\Delta \beta \\
\Delta \alpha
\end{array}\right]=\left[\begin{array}{c}
-c_{M y}^{i-1} \\
c_{D, \text { target }}^{i}-c_{D}^{i-1} \\
c_{L, \text { target }}-c_{L}^{i-1}
\end{array}\right]} \\
c_{D, \text { target }}^{i \text { def }}=\sin \gamma c_{L}^{i-1}
\end{gathered}
$$

\subsection{Calculation of dynamic derivatives}

For the calculation of the dynamic derivatives, forced harmonic pitch motions are simulated with the flow solver TAU. Assuming that the aerodynamic coefficients are linear functions of the angle of attack, the temporal change in angle of attack, the pitching angular velocity and the angular acceleration, their differences with respect to the mean values can be written as eq.3. With the definition of the forced harmonic motion (eq. 4), the time dependent variables $\dot{\alpha}, q$ and $\dot{q}$ can be written as eq. 5 . The derivatives of the aerodynamic coefficients can then be derived by Fourier analysis or least square regression of the resulting time series of the aerodynamic coefficients.

$$
\begin{gathered}
\Delta c_{i}=c_{i \alpha} \Delta \alpha+c_{i \dot{\alpha}} \frac{c_{r e f}}{V_{\infty}} \dot{\alpha}+c_{i q} \frac{c_{r e f}}{V_{\infty}} q+c_{i \dot{q}} \frac{c_{r e f}^{2}}{V_{\infty}^{2}} \dot{q} \\
\Delta \alpha=\alpha_{A} \sin (\omega t)
\end{gathered}
$$




$$
\begin{gathered}
\dot{\alpha}=q=\alpha_{A} \omega \cos (\omega t) \\
\dot{q}=-\alpha_{A} \omega^{2} \sin (\omega t)
\end{gathered}
$$

\section{GEOMETRY}

The basis of the investigations is a generic medium range transport aircraft, which was designed with the preliminary aircraft design tool PrADO [20] in the course of the CRC 880. With maximum payload corresponding to 100 passengers, the mission range is around $2000 \mathrm{~km}$ at a cruise Mach number of $\mathrm{Ma}_{\mathrm{cr}}=0.74$. In order to achieve the targeted maximum take-off and landing distance of less than $800 \mathrm{~m}$, the aircraft is powered by turboprop engines and equipped with internally blown plain flaps and ailerons. Blowing is realized by the integration of a high-pressure plenum into the main wing above the flap kink, which is located at a relative chord length of $\mathrm{c}_{\text {Kink }} / \mathrm{c}=0.75$ (Fig. 1). The aerodynamic influence of the flap length was investigated in an earlier study [21]. While longer flap lengths lead to higher lift coefficients, they also reduce the lift gain factor, i.e. the efficiency of the circulation control. Furthermore, longer flap lengths reduce the possible wing tank volume and can have a negative impact on structure weight.

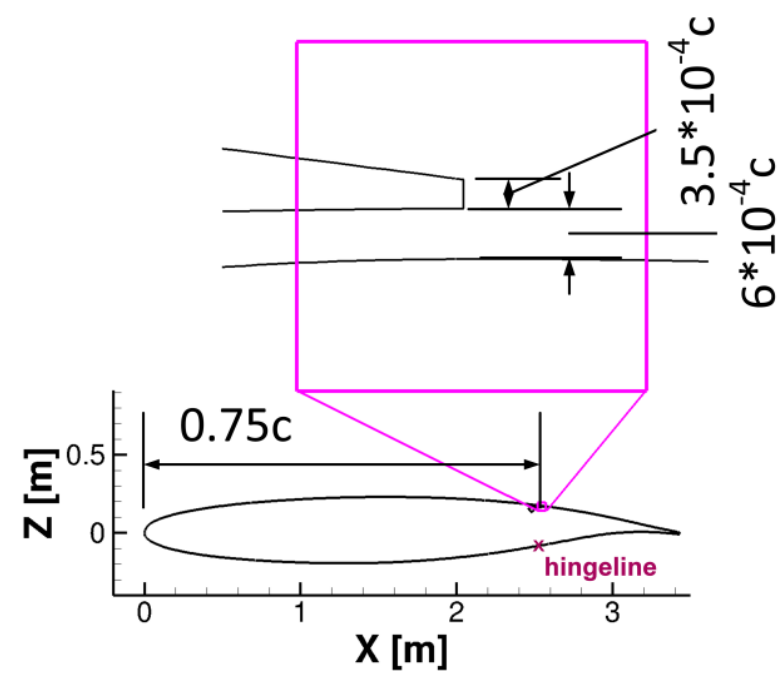

Figure 1 F15 airfoil with retracted flap and blowing slot geometry

The first simulations of the investigation were performed with the landing configuration of the initial aircraft design REF0-2011. In this configuration, the internally blown flap is deflected by $\delta_{\text {Flap }}=65^{\circ}$ and the aileron is drooped by $\delta_{\text {Aileron }}=$ $45^{\circ}$. However, the baseline aircraft does not utilize a leading edge device. For optimal efficiency of the circulation control, the plenum along the wing span is separated into six sections, which can be independently pressurized to adjust the blowing to the local flow conditions [22]. For the investigation of the lateral motion and the failure cases, the total pressure within the plena was tuned to obtain fully attached flaps and ailerons at the smallest possible amount of blowing. At an angle of attack of $\alpha=0.0^{\circ}$, the resulting global blowing coefficient equates to $\mathrm{c}_{\mu}=0.033$.

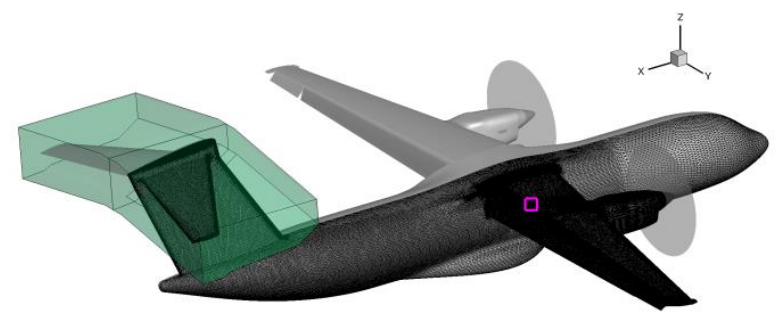

a) Geometry overview with module box and location of mesh detail

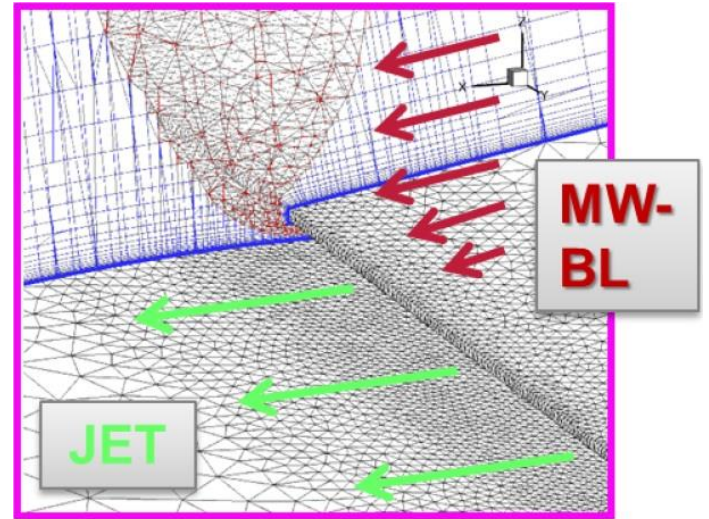

b) mesh detail

Figure 2 Geometry and CFD mesh (REF2-2013)

As the RANS computations revealed, the initial aircraft design had several drawbacks, which were addressed by a PrADO design update. As a result, the size of the vertical as well as the horizontal tail plane of the new design REF2-2013 is increased. Furthermore, the aircraft's main wing is tilted by $\Delta \mathrm{i}_{\mathrm{MW}}=-3.44^{\circ}$ and it utilizes a smart droop nose in high-lift configuration in order to allow for higher angles of attack. The design update also resulted in an increased nacelle.

The hybrid CFD meshes were built with the semiautomated mesh generator Centaur. The mesh was set up modularly, in order to allow a wide range of HTP trim angles (Fig. 2a). However, during the trim procedure, the change in HTP deflection is sufficiently small to use mesh deformation. The most critical part of the mesh generation process is the adequate resolution of the region of the jet exit slot. In contrast to the dimension of the full aircraft and the rather low free 


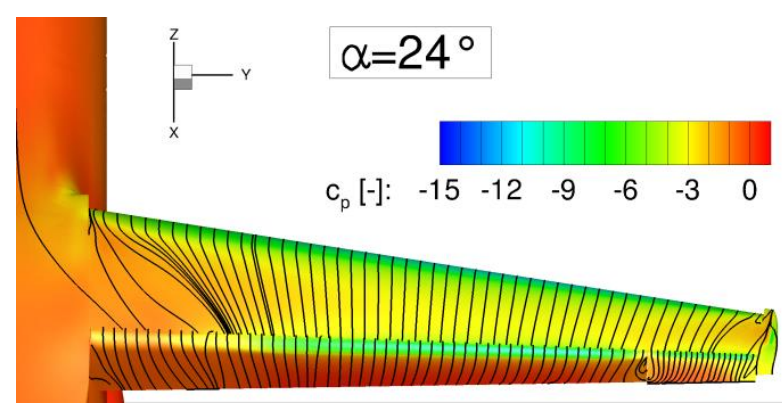

a) without nacelle

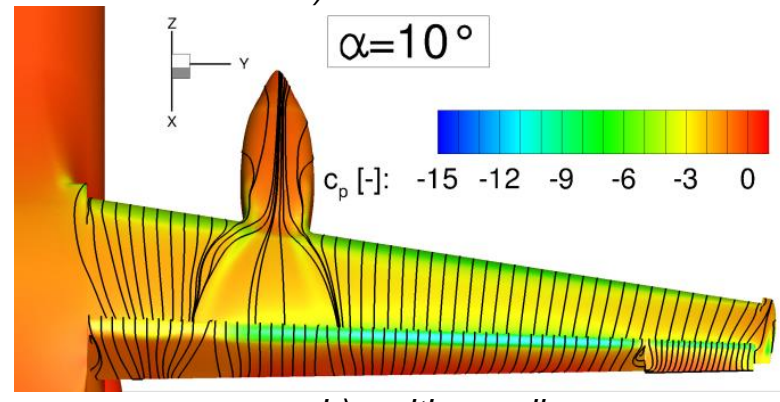

b) with nacelle

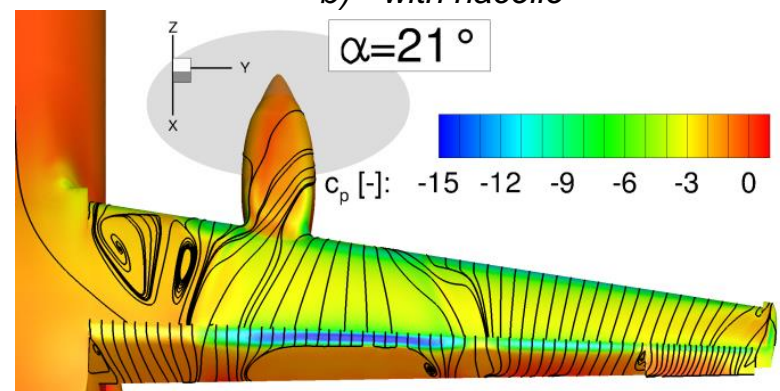

c) with nacelle and thrust

Figure 3 Variation of stall behavior at $c_{\mu}=0.03$ visualized by pressure distribution and skin friction lines at post-stall angle of attack

stream Mach number of $\mathrm{Ma}_{\infty}=0.15$, the trailing edge thickness above the slot becomes as small as $t_{T E}=0.03 \% c_{R E F}$ in the outboard region. Furthermore, the local Mach number at the slot exit can be close to $\mathrm{Ma}=1$ resulting in a low first layer height of the prisms. As a consequence of this, the mesh size of the half model, which is used for the analysis of the longitudinal motion, ranges between $37.5 \mathrm{M}$ and $48.5 \mathrm{M}$ points for REF0-2011 and REF2-2013, respectively.

\section{RESULTS}

\subsection{Stall Behavior}

The stall behavior of a wing in high-lift configuration is affected by various aspects, such as wing geometry, high-lift devices, wing-bodyinteraction and engine airframe interference effects. Especially, for wing-mounted turboprop engines, the latter can have a considerable influence. First, the nacelle disturbs the homogenously oncoming flow, as it acts as an additional lifting body in front of the wing and furthermore the nacelle contour is intersecting with the wing and its high lift devices. Second, the propeller slipstream interferes with the main wing, resulting in increased velocities of the oncoming flow and altered local angles of attack.

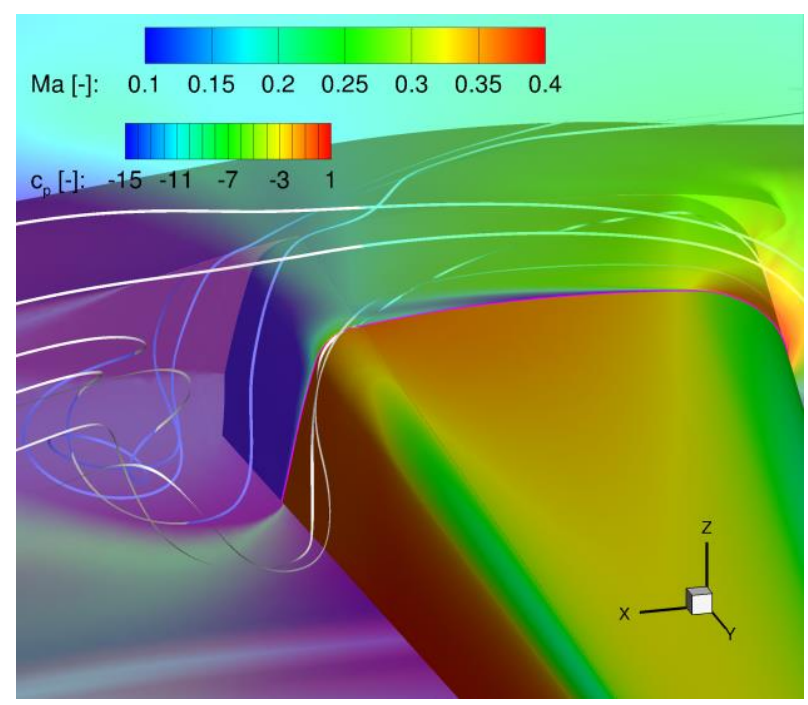

Figure 4 Distorted flow conditions in the wingbody junction at $=24^{\circ}, c_{\mu}=0.03$ and no nacelle and position of Y-cutting plane (Fig. 5)

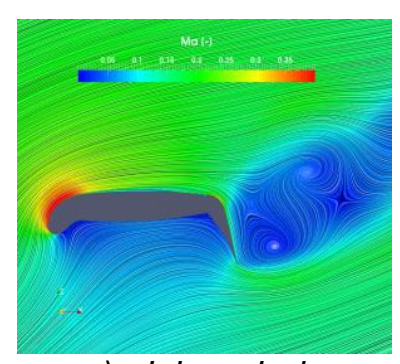

a) Inboard wing section

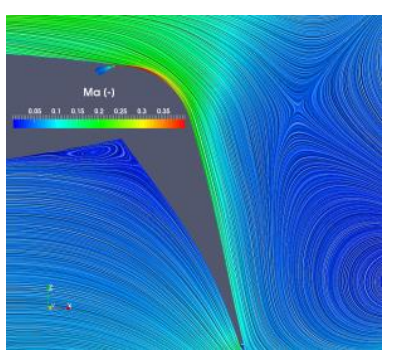

b) Flap region
Figure 5 Mach number distribution and in-plane flow directions in $Y$-cutting plane at $\alpha=24^{\circ}, c_{\mu}=$ 0.03 and no nacelle

Regarding the configuration under investigation, the impact of the engines on the stall behavior is significant, as can be seen in the isobars and wall streamlines in Fig. 3. Related CFD computations reveal that at a blowing coefficient of $c_{\mu}=0.03$ and without nacelle, the main wing stalls beyond $\alpha_{\max }=23^{\circ}$ due to strong cross flow on the inboard part of the wing. While the CC jet and parts of the main wing boundary layer can follow the flap contour, the low momentum boundary layer, coming from the fuselage, leads to a recirculation region behind the flap and in consequence to a velocity decrease and pressure increase (Fig. 4). As a result, the outer part of the main wing 
boundary layer separates from the flap (Fig. 5). The resulting surface pressure increase (Fig. 3a) on the upper side of the flap triggers a strong lift break down on the inboard part of the wing.

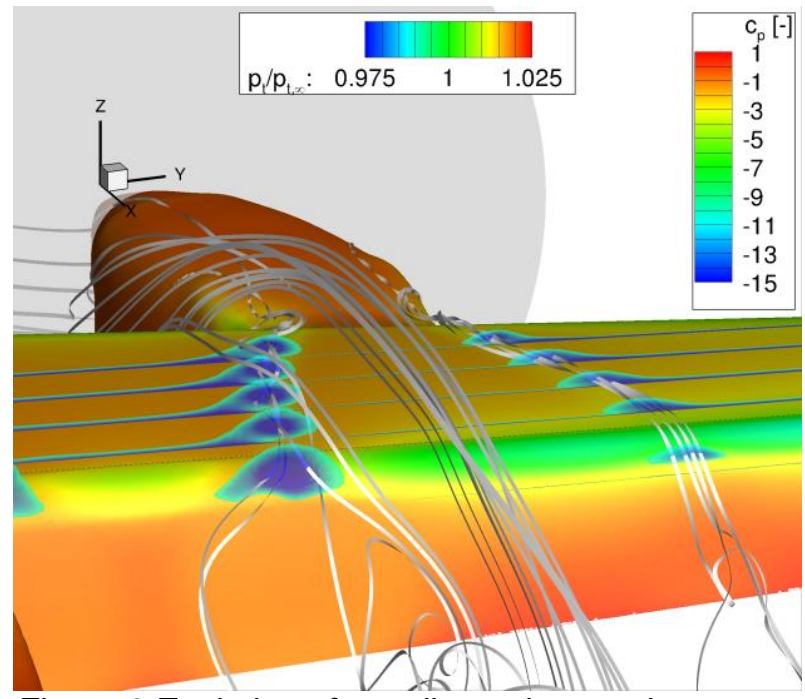

Figure 6 Evolution of nacelle vortices and resulting total pressure loss at $\alpha=10^{\circ}, c_{\mu}=$ 0.03 and no thrust

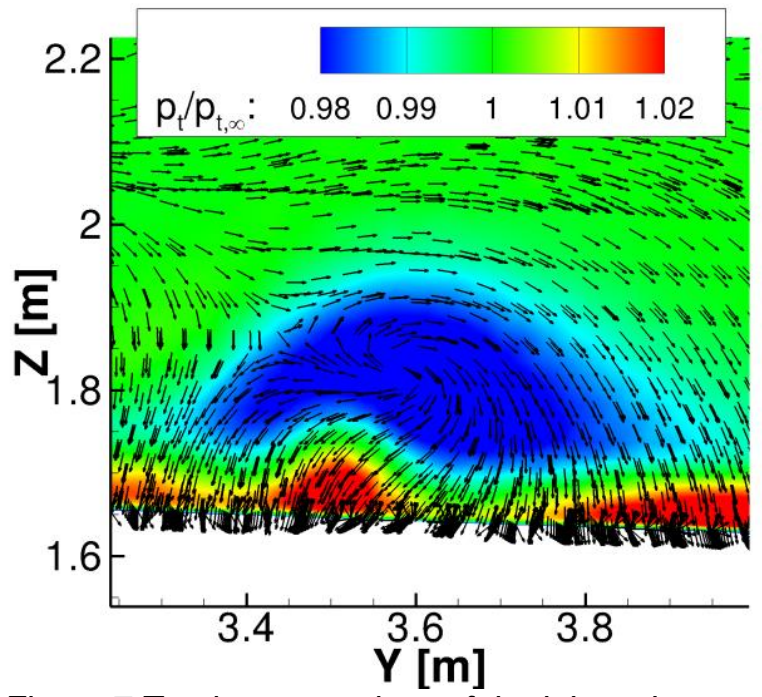

Figure 7 Total pressure loss of the inboard nacelle vortex and total pressure reduction within the blowing jet at $\alpha=8^{\circ}, c_{\mu}=0.03$ and no thrust

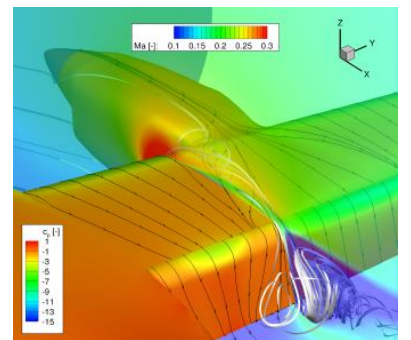

a) without strake

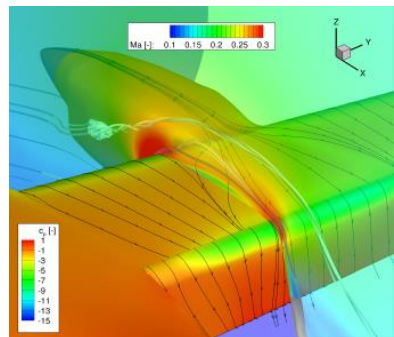

b) with strake
Figure 8 Comparison of flow behind nacelle at $\alpha=10^{\circ}$ and $c_{\mu}=0.03$
If nacelles are added without thrust generation, the maximum angle of attack is severely reduced to $\alpha_{\max }=7^{\circ}$ and the stall mechanism is completely changed as can be seen for the post stall condition in Fig. 3b. Maximum lift is now limited by the inboard nacelle vortex. Due to the aircraft's angle of attack and the high main wing circulation, the nacelle is exposed to a considerable local angle of attack. As a result, nacelle vortices roll up and weaken the main wing boundary layer, with the inboard nacelle vortex being the more critical flow feature due to the wing sweep (Fig. 6). In addition to the total pressure loss within the main wing boundary layer, the nacelle vortices also disturb the CC jet above the flap. Typically, the downwash side of a vortex close to a wall is the less critical for the boundary layer regarding separation, since it tends to reenergize the boundary layer with higher momentum flow from the outer flow. However, in this case, the jet has more momentum than the outer flow. As a result, the flow on the downwash side of the vortex appears to be the more critical aspect for the blowing jet. To prove this observation, Fig. 7 shows how the excess momentum within the jet is deteriorated by the inboard nacelle vortex on the downwash side. Even though, the blowing jet is fully attached to the surface even for post-stall condition $\left(\alpha=10^{\circ}\right)$, it is assumed that these two effects, the total pressure loss within the main wing boundary layer and the total pressure reduction within the blowing jet, lead to an reduced velocity and increased pressure above the flap, which in turn results in a wake/vortex burst above the flap (Fig. 8a). It cannot be fully ruled out that the vortex burst is the initial event, which simply occurs due to the positive pressure gradient above the flap. However, the nacelle vortex strength increases with rising angle of attack and is therefore less prone to a vortex burst. Furthermore, the flap pressure gradient is rather coupled to the blowing coefficient and stays almost constant with rising angle of attack. Therefore, it is assumed to be less likely that the vortex burst at higher angles of attack occurs without the weakening of the main wing boundary layer and the blowing jet.

Applying thrust and slipstream to the flowfield increases the maximum angle of attack again. The propeller leads to higher flow velocities at the nacelle, which in turn lead to a smaller local angle of attack, even on the upwash side of the propeller, compared to the case without thrust. Thus, the nacelle vortices are reduced in strength. Furthermore, the additional momentum lowers the total pressure loss (with respect to the free stream total pressure) within the vortices. As a result, the thrust effects alleviate the nacelle vortex impact so 
that the lift break down is shifted from downstream of the nacelle to the inboard part of the wing (see Fig. $3 c$ ). On the outboard side of the nacelle, the flow is separated on the flap. However, the flap suction peak is increased in this region. Both effects can be attributed to the propeller slipstream and are present for all investigated angles of attack. Thus, they are not the cause of the stall. With increasing angle of attack $\left(\alpha=18^{\circ}\right)$, the offsurface separation, observed in the case without nacelle occurs in proximity to the fuselage and reduces the local circulation. Yet, the lift of the entire wing continues to rise (see Fig. 9, w/o strake | $w /$ thrust). Maximum lift is imposed by an inboard leading edge separation. It can be concluded that the cross flow coming from the fuselage in addition to the increased oncoming flow at increased local angle of attack due to the propeller leads to the separation. However, the maximum angle of attack is reached at $\alpha_{\max }=20^{\circ}$ and is therefore only slightly lower than the one of the wing without nacelle. Since the stall occurs due to a separation in the upwash region of the propeller, the maximum angle of attack is certainly dependent on the accuracy of the actuator disk model. However, comparisons of simulations with actuator disk model with wind-tunnel tests delivered good results, especially regarding the flow conditions behind the Propeller $[17,18]$.

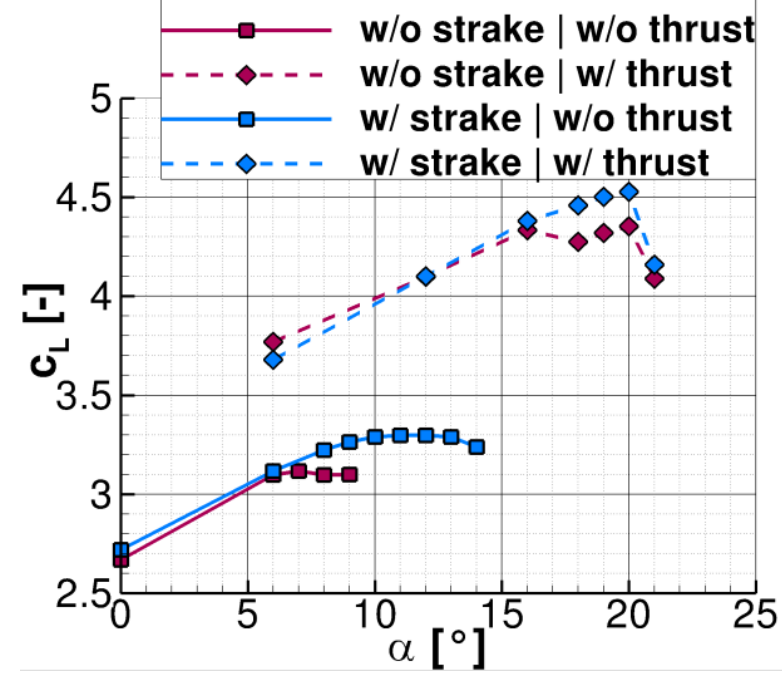

Figure 9 Influence of strake on lift curve with and without thrust

The most critical case is the one with nacelle and without thrust. Therefore, it was concluded to design a nacelle strake, which aims at altering the nacelle vortex strength and thus improving the stall behavior especially for this case.

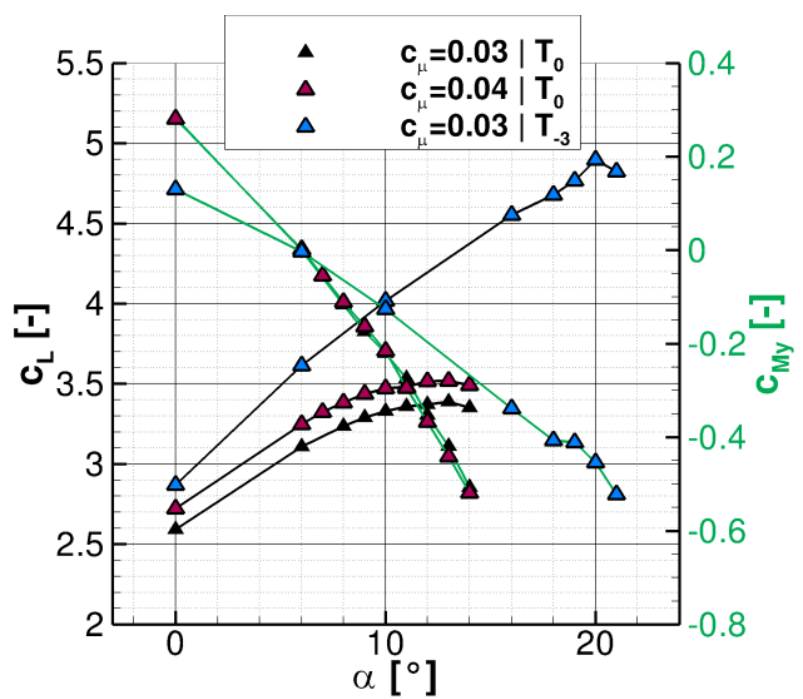

Figure 10 Lift and pitching moment curves for various blowing and thrust settings

First results of the configuration with strake already show an improvement of the flow conditions behind the nacelle (Fig. 8). If adequately positioned, the resulting strake vortex suppresses the inboard nacelle vortex and leads to an attached flow on the flap even at higher angles of attack. As a result, the maximum angle of attack is enlarged by several degrees in case of zero thrust (Fig. 9). Furthermore, the maximum lift coefficient is increased by $\Delta \mathrm{C}_{\mathrm{L}, \mathrm{w} / \mathrm{o} \text { thrust }}=0.18$. At a moderate thrust setting of $\mathrm{T}=49 \mathrm{kN}\left(\mathrm{C}_{\mathrm{T}}=0.24\right)$, the maximum lift augmentation of the wing-body is comparable with $\Delta \mathrm{C}_{\mathrm{L}, \mathrm{w} / \text { thrust }}=0.18$. However, the maximum angle of attack remains unaffected.

\subsection{Longitudinal Static Behavior}

For the investigation of the longitudinal static stability of REF2-2013, blowing as well as thrust has been varied. The aircraft was specifically trimmed for the alternating settings with respect to the center of gravity position at maximum landing weight with maximum payload. For the case with thrust, the pitch angle of the propeller blades was trimmed in order to achieve a balanced flight at a glide slope of $y=-3^{\circ}$. For the calculation of the angle of attack variation, the thrust was then kept constant. Tab. 1 shows the HTP trim angles for the different settings.

Table 1 HTP trim angles for different blowing and thrust settings

\begin{tabular}{|c|c|c|c|c|}
\hline Case & $\mathrm{C}_{\mu}$ & $\mathrm{C}_{\mathrm{T}}$ & $\mathrm{C}_{\mathrm{L}, \mathrm{MW}+\text { Prop }}$ & $\mathrm{i}_{\mathrm{HTP}}$ \\
\hline $\mathrm{C}_{\mu}=0.03 \mid \mathrm{T}_{0}$ & 0.03 & 0 & 3.11 & $3.46^{\circ}$ \\
\hline $\mathrm{C}_{\mu}=0.04 \mid \mathrm{T}_{0}$ & 0.04 & 0 & 3.26 & $3.87^{\circ}$ \\
\hline $\mathrm{C}_{\mu}=0.03 \mid \mathrm{T}_{-3}$ & 0.03 & 0.54 & 3.56 & $4.78^{\circ}$ \\
\hline
\end{tabular}




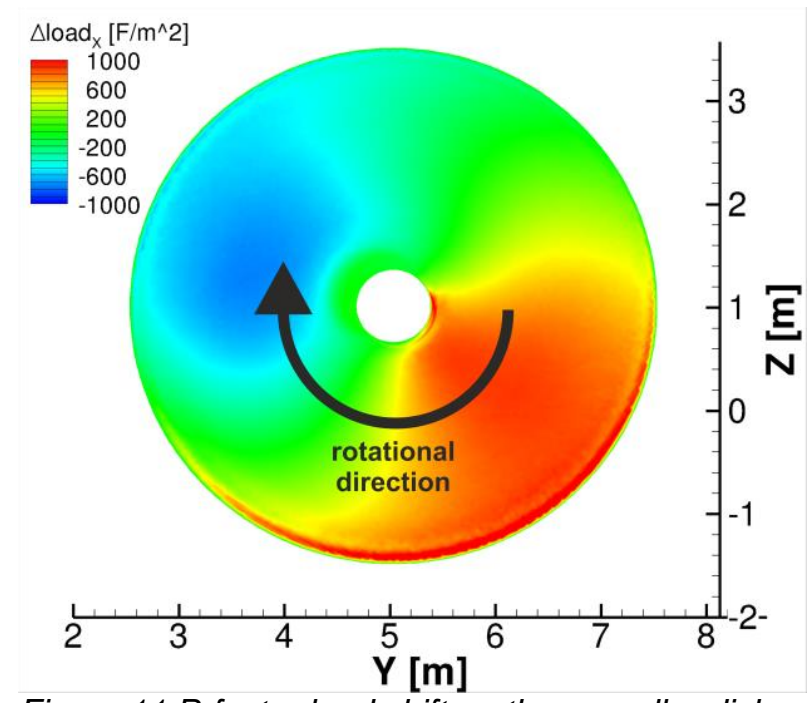

Figure $11 P$-factor load shift on the propeller disk between $\alpha=10^{\circ}$ and $\alpha=0^{\circ}$ a) Wing-Body and Prop

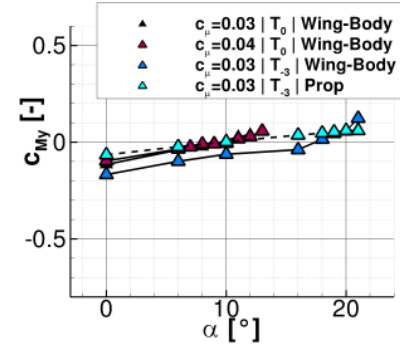

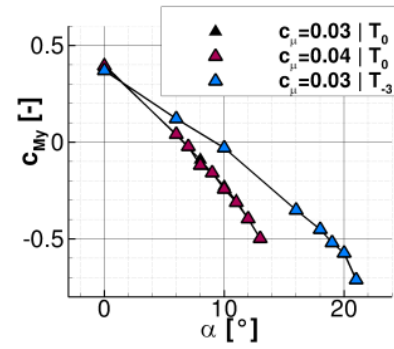

b) HTP
Figure 12 Detailed pitching moment curves dependent on angle of attack

Fig. 10 depicts the lift coefficients of the entire aircraft including propeller thrust dependent on the angle of attack. Increased blowing leads to an upward shift of the lift curve. The lift slope does not change with altering blowing. When regarding an isolated wing with high flap deflection and circulation control, increased blowing generally also leads to a reduced maximum angle of attack, since the critical adverse pressure gradient of the main wing boundary layer is reached earlier due to a stronger leading edge suction peak. However, for the integrated case, the maximum angle of attack is not limited by airfoil characteristics but by the influence of the nacelle. As a result, the sensitivity of the maximum angle of attack with respect to the blowing coefficient is not visible, here. Due to the additional force of the propeller, the thrust leads to an increase in the lift slope. Furthermore, the lift of the main wing is raised by the locally increased velocity of the oncoming flow within the slipstream, resulting in a higher lift slope and higher $\mathrm{C}_{\mathrm{L}\left(\mathrm{\alpha}=0^{\circ}\right)}$. As discussed in section 3.1, the maximum angle of attack is also raised.
The pitching moment curves of all investigated cases have a negative slope, demonstrating a stable behavior for the investigated center gravity. However, while the pitching moment distribution is nearly unaffected by a change in blowing, the steepness of the slope is reduced due to thrust. With rising angle of attack, the propeller load is shifted downwards due to the $p$ factor (Fig. 11), creating an additional positive

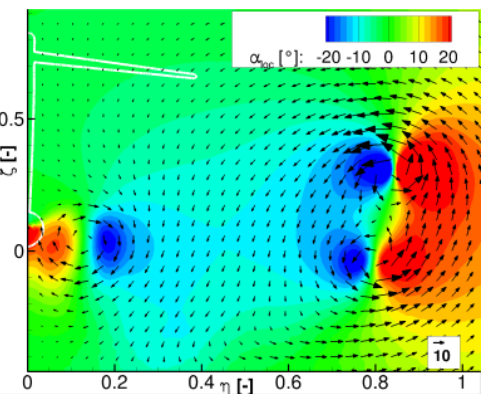
a) $c_{\mu}=0.03, T_{0}$

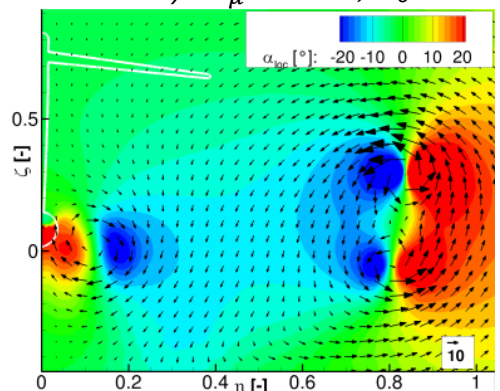

b) $c_{\mu}=0.04, T_{0}$

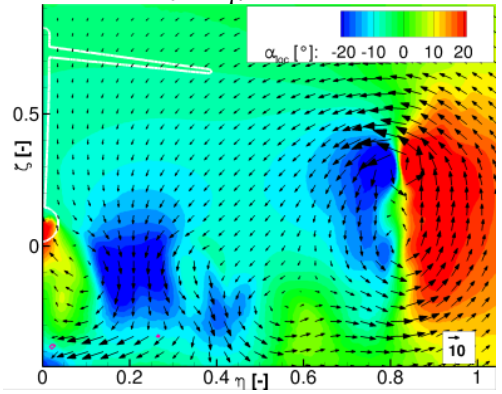

c) $c_{\mu}=0.03, T_{-3}$

Figure 13 Flow conditions at the HTP position at $\alpha=6^{\circ}$ pitching moment gradient (Fig. 12a). Furthermore, the main wing load is changed by the slipstream, leading to a stronger downwash below the HTP [10] (Fig. 13). As a result, the vertical gradients of the local angle of attack are stronger, which in turn reduces the dampening effect of the HTP (Fig. 12b).

The result is a movement of the stability limit towards the front. At zero thrust, the stability limit is located at $\frac{\Delta X_{\text {stab, }} \mathrm{c}_{\mu=0.03, \mathrm{~T}_{0}}}{c_{\text {ref }}}=0.67 \quad$ and $\frac{\Delta X_{\text {stab }, c_{\mu=0.04, T_{0}}}}{c_{\text {ref }}}=0.65$ for $\mathrm{c}_{\mu}=0.03$ and $\mathrm{c}_{\mu}=0.04$, respectively. With thrust, it moves to $\frac{\Delta X_{\text {stab }, c_{\mu=0.03, \mathrm{~T}_{1}}}}{c_{\text {ref }}}=0.34$ at $\mathrm{c}_{\mu}=0.03$.

\subsection{Longitudinal Dynamic Behavior}

Prior to the three-dimensional investigations of the dynamic behavior, a parametric study of a representative two-dimensional geometry was performed. Besides algorithmic parameters, also 
parameters regarding the configuration and boundary conditions were examined. Assuming that three-dimensional effects do play a role, but show similar effects for different parameter values, the 2D-study can give an indication of the general trends with respect to selected parameters. In contrast, the 3D simulations give more accurate results for only few selected operating points due to their high computational costs.

Fig. 14 shows the pitch damping derivative of the main airfoil depending on flap deflection, blowing coefficient and angle of attack. It shows that the

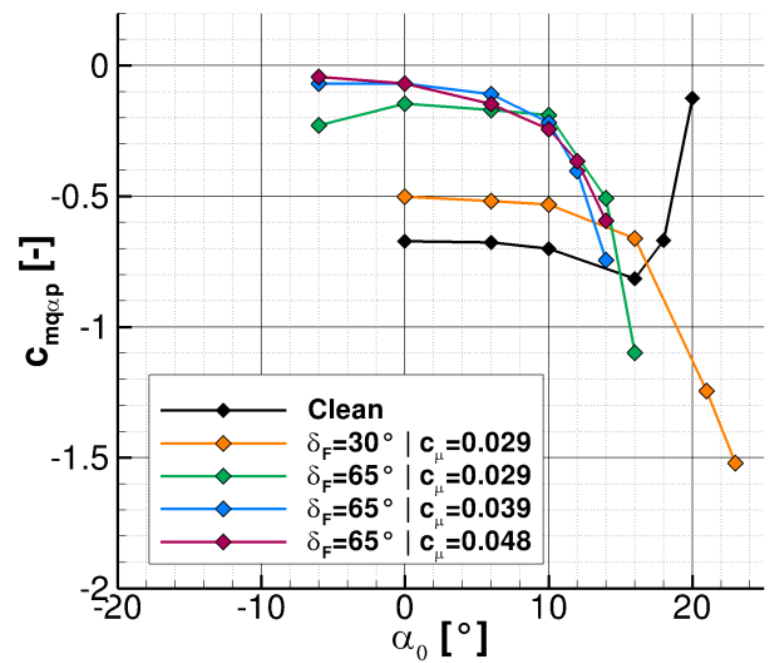

Figure 15. Variation of the main airfoil's pitch damping derivative with respect to flap deflection, blowing coefficient and angle of attack

damping is reduced if the flap deflection is increased. Whether circulation control works as boundary layer control or in super circulation mode plays a minor role. Furthermore, the pitch damping derivative only slightly changes with the angle of attack in the linear region. However, close to the maximum angle of attack, the cases with flap deflection show a steep increase in magnitude, whereas the case without high-lift devices ("Clean") shows the opposite trend. This difference can be explained with the varying stall mechanism.

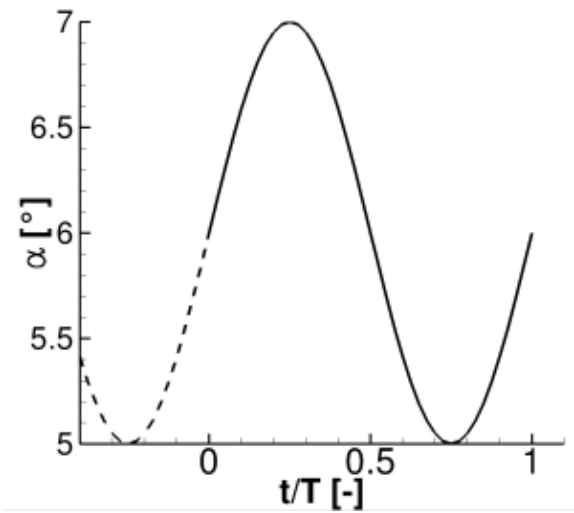

Figure 16 Forced pitching motion (evaluation period)

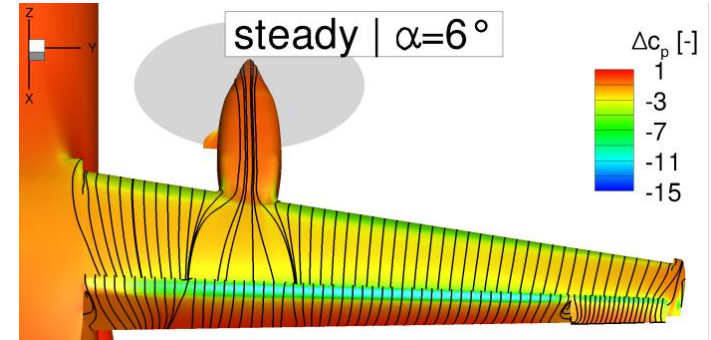

Figure 14 Steady state pressure distribution and skin friction lines of the main wing at $c_{\mu}=0.03$ and $\alpha=6^{\circ}$

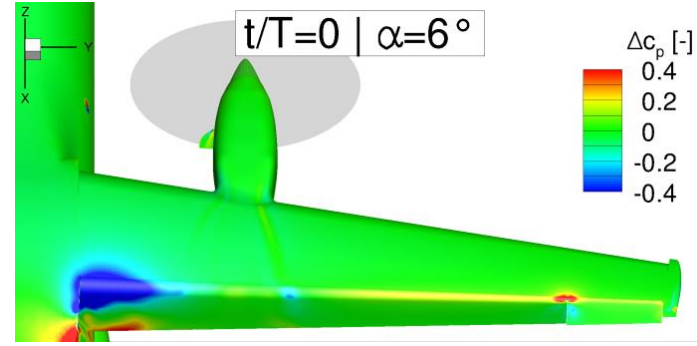

Figure 17 Difference in pressure distribution due to pitch up motion

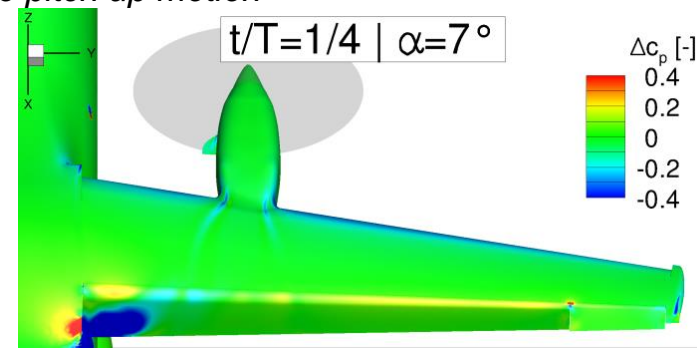

Figure 18 Difference in pressure distribution at $t / T=1 / 4$

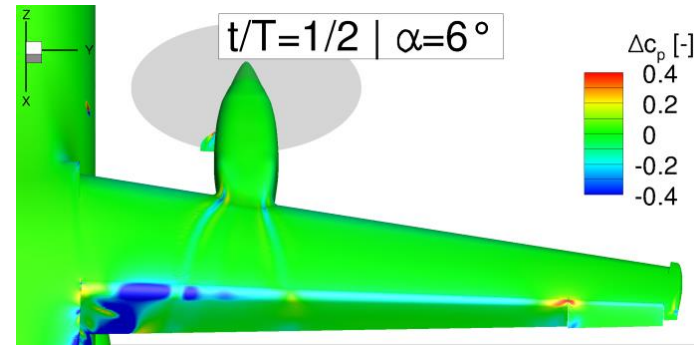

Figure 19 Difference in pressure distribution due to pitch down motion

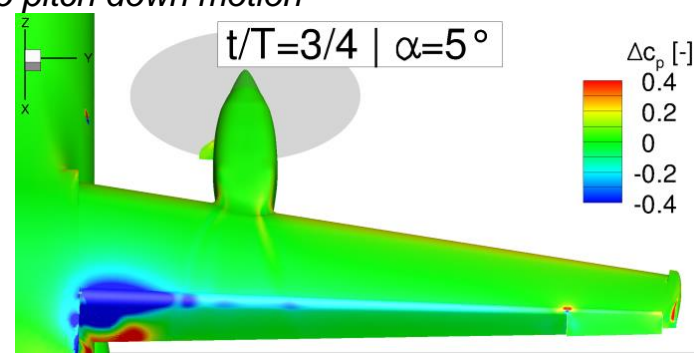

Figure 20 Difference in pressure distribution at $t / T=3 / 4$

The calculation of the dynamic derivatives of the REF2-2013 model were performed with strake at a blowing coefficient of $\mathrm{C}_{\mu}=0.03$, zero thrust and an angle of attack of $\alpha=6^{\circ}$. Based on the 2D study, a 
reduced frequency of $\mathrm{k}=1$ and an amplitude of $\Delta \alpha$ $=1^{\circ}$ was selected for the $3 \mathrm{D}$ simulation of the pitching motion (Fig. 15). These parameter values result in an equivalent angular velocity of $q=15 \%$ at reference conditions. Fig. 16 shows the pressure distribution and the skin friction lines of the main wing for the reference conditions at steady state. It shows that the flow is completely attached to the wing, even though the flow is distracted to the sides behind the nacelle due to the nacelle vortices.

Fig. 17 depicts the difference in pressure distribution between the pitch up motion at maximum angular velocity and the steady state. The biggest impact can be seen in the rear part of the inboard region. Close to the fuselage, a recirculation area exists behind the flap. Due to the pitching motion, the local velocity is increased, which in turn changes the surface pressure distribution on the flap. Therefore, the suction peak on the flap is increased. Behind the nacelle, one can locate the trace of the nacelle vortices. At steady state, they are close to the surface and due to their low pressure level within the vortex core the surface pressure is slightly lower below the vortices. However, their distortion of the boundary layer negatively affects the flow on the flap, where the suction peak is reduced. When the aircraft pitches up, the pressure below the vortices is increased on the main wing and decreased on the flap. A possible cause might be a slight change of the vortex strength, due to the motion. Furthermore, the locations of the vortices change with respect to the main wing.

On the outboard wing, the flap suction peak is slightly reduced. Furthermore, changes are visible in close proximity to the flap and aileron side vortices.

At $t / T=1 / 4$ (Fig. 18), the harmonic motion reaches the highest angle of attack. As a result, the leading edge suction peak is increased and the flap suction peak is decreased, as it is already known from the static behavior, when the angle of attack is increased. Additionally, a pressure reduction can be identified at the trailing edge of the flap close to the fuselage, which indicates, in conjunction with the reduced flap suction peak, a local separation.

Fig. 19 shows the difference in pressure distribution for the maximum negative angular velocity. Again, the biggest impacts are visible at the side ends of flap and aileron. Analogously to the upward motion, the suction peak of the flap is now increased in the outboard region. Furthermore, the surface pressure below the nacelle vortices is reduced on the main wing and increased on the flap due to the closer distance of the vortices to the surface.
At the lower turning point of the pitching oscillation (Fig. 20), the leading edge suction peak is reduced and the flap suction peak is increased due to the lower angle of attack. The strongest effect can be seen at the fuselage-flap junction, where the pressure at the trailing edge as well as the flap suction peak is increased. This leads to the assumption that the flow is better following the contour. Flow visualizations of the relative velocities with respect to the moving aircraft confirm this assumption.

The strong variation of the surface pressure at the location of the inboard flap vortex leads to a nonlinear behavior of the main wing's lift, as seen in Fig. 21. The maximum lift is reached before the upper turning point is reached. Analogously, the lift coefficient rises already, even though the aircraft is still pitching downward. As a result, the assumption of linear behavior does not lead to a good regression.

However, the regression fits better for the full aircraft coefficients due to the influence of the HTP (Fig. 22). Especially, the pitching moment is represented well (Fig. 23), as the HTP plays a dominating role, here.

Evaluating the dynamic derivatives based on the least square regression leads to a lift derivative of $c_{L q+L \dot{\alpha}}=6.4$ and a pitch damping derivative of $c_{M y q+M y \dot{\alpha}}=-23.3$. The calculation of the dynamic derivatives of the corresponding $2 \mathrm{D}$ case results in a lift derivative of $c_{l q+l \dot{\alpha}}=3.3$ and a pitch damping derivative of $c_{m y q+m y \dot{\alpha}}=-21.5$. Thus, the results of the representative $2 \mathrm{D}$ simulation do not seem to give a good estimation regarding lift. The difference can be traced back to the main wing contribution. While the lifting force derivative of the HTP differs only slightly between $3 \mathrm{D}\left(c_{L q+L \dot{\alpha}, H T P}=\right.$ 4.65) and $2 \mathrm{D}\left(c_{L q+L \dot{\alpha}, H T P}=4.63\right)$, it shows a large mismatch for the main wing $c_{L q+L \dot{\alpha}, M W}=1.78$ (3D) versus $c_{L q+L \dot{\alpha}, M W}=-1.37(2 \mathrm{D})$. Since the center of rotation is close to the main wing, the dynamic derivatives of the main wing are more sensitive to a change of the center of rotation. In order to determine the center of rotation for the 2D simulations, the geometric aerodynamic center of the $3 \mathrm{D}$ configuration was used. As a result, the $2 \mathrm{D}$ geometry does not represent the exactly correct relative positions between the airfoils and the center of rotation. Furthermore, the representation of the main wing by the main wing airfoil is worse compared to the HTP, due to spanwise geometry variations including engine and aileron. However, the 2D simulations give a good indication of the pitch damping derivative, which only differs by $8 \%$ from the 3D results, since it is, once again, dominated by the HTP. 


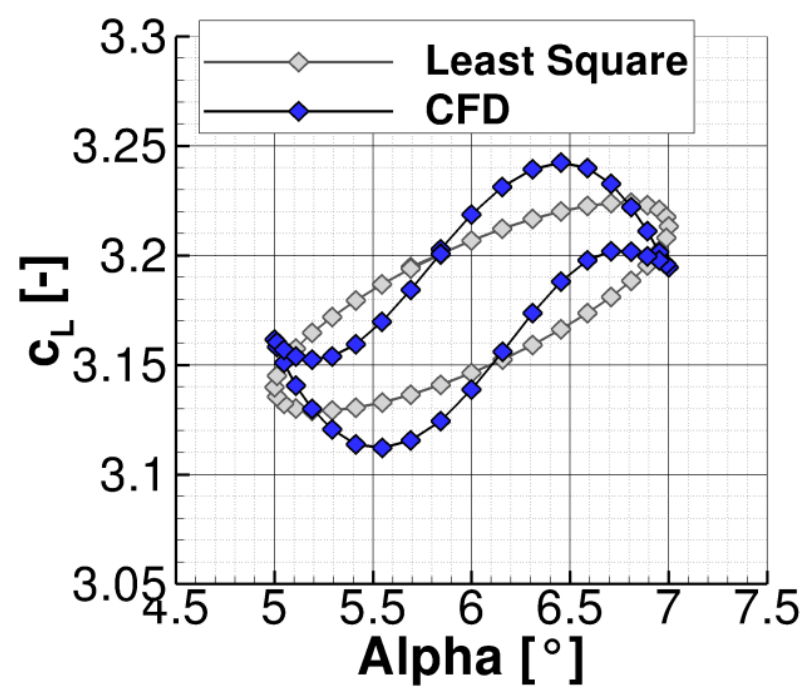

Figure 21 Wing-body lift coefficient from RANS calculation and least square fit for the pitching oscillation

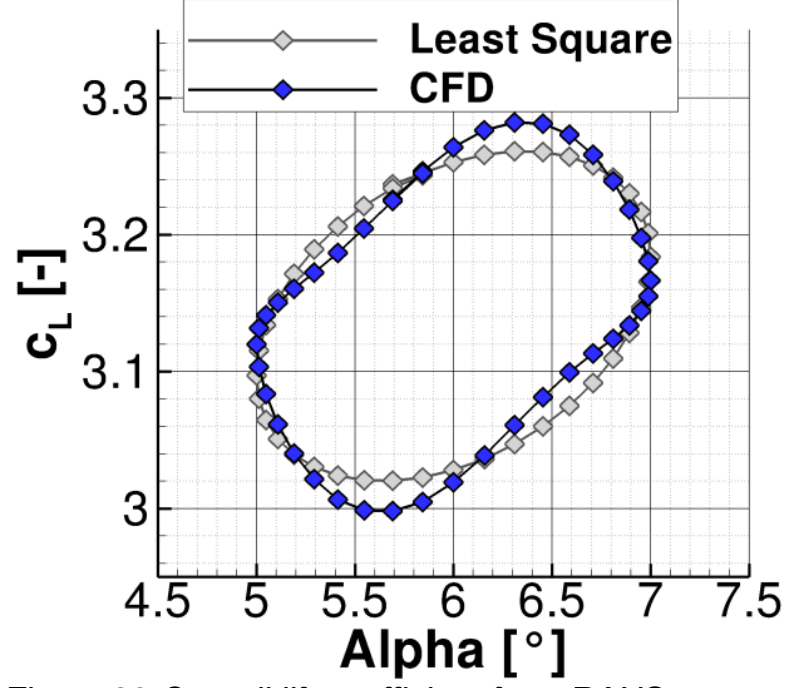

Figure 22 Overall lift coefficient from RANS calculation and least square fit for the pitching oscillation

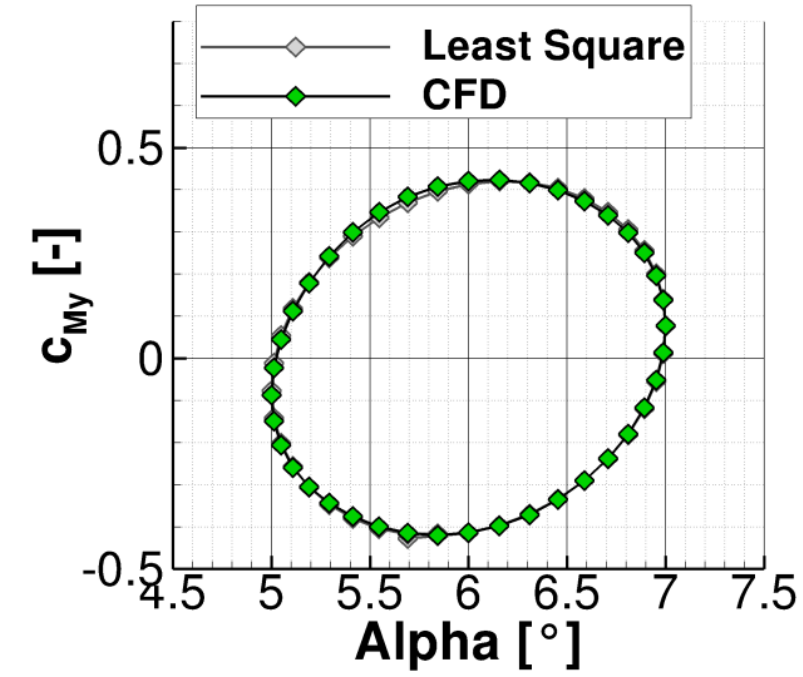

Figure 23 Overall pitching moment coefficient from RANS calculation and least square fit for the pitching oscillation

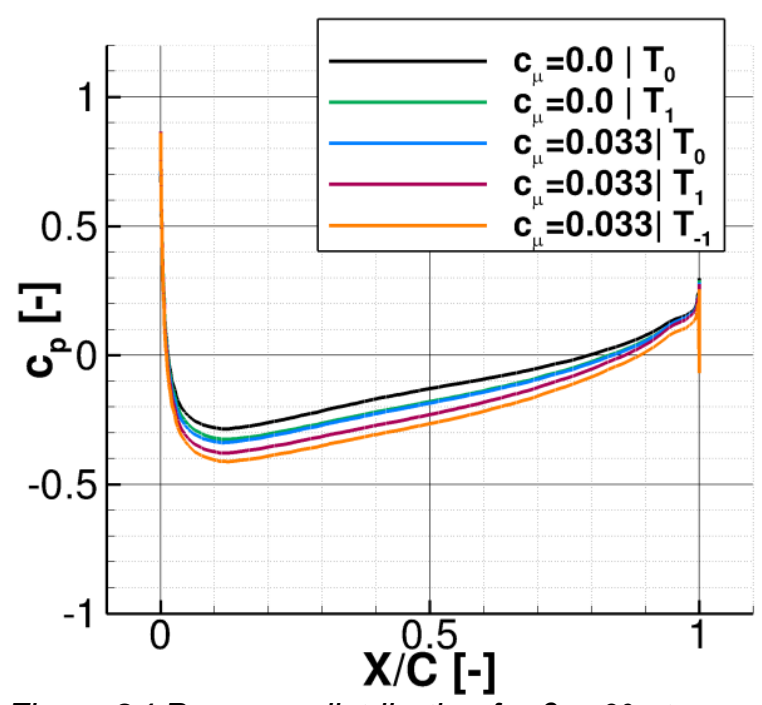

Figure 24 Pressure distribution for $\beta=0^{\circ}$ at $\eta_{V T P}$ $=0.45$

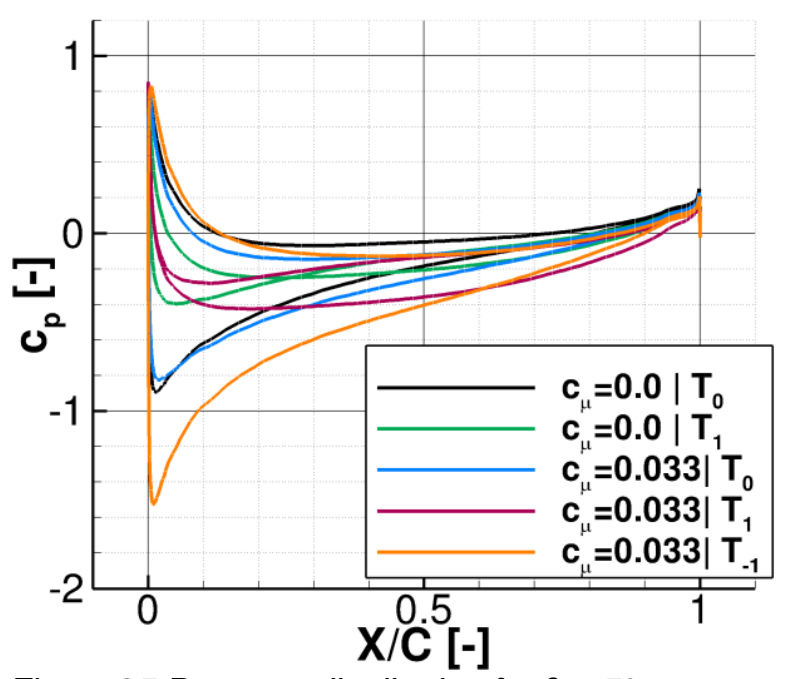

Figure 25 Pressure distribution for $\beta=5^{\circ}$ at $\eta_{V T P}$ $=0.45$ 


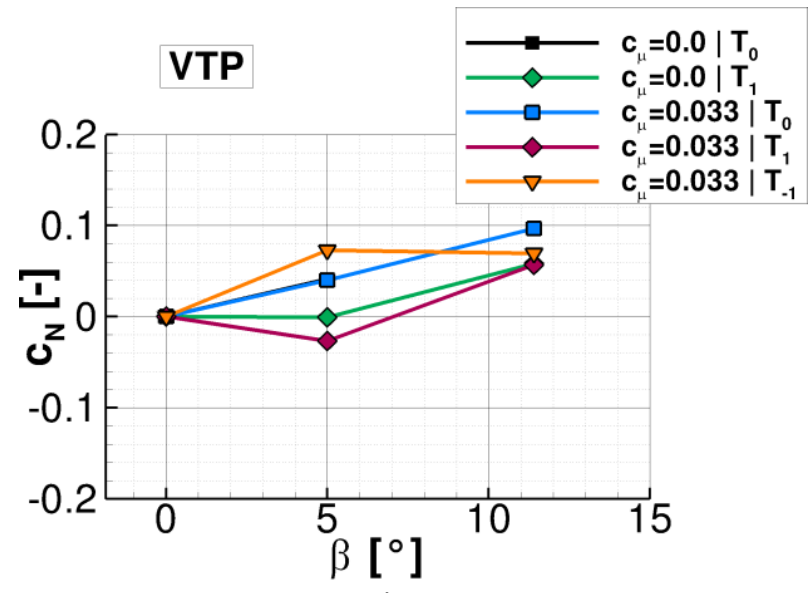

a) VTP

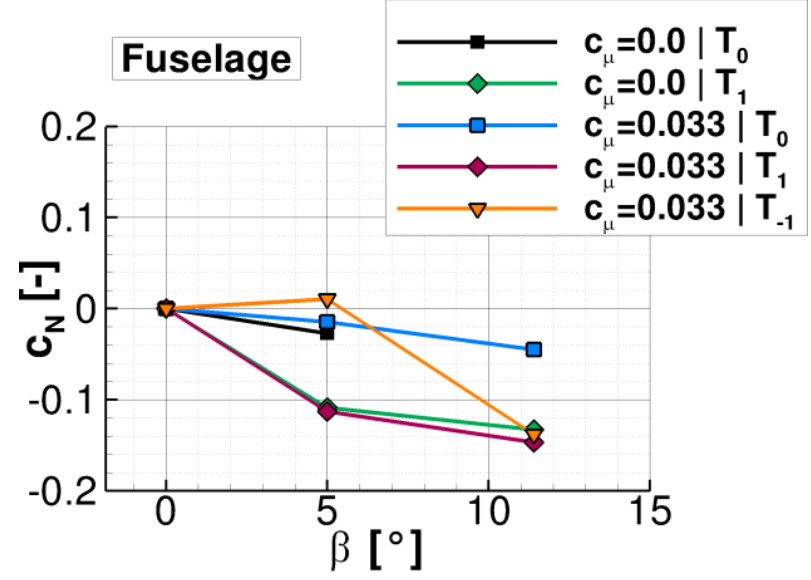

b) Fuselage

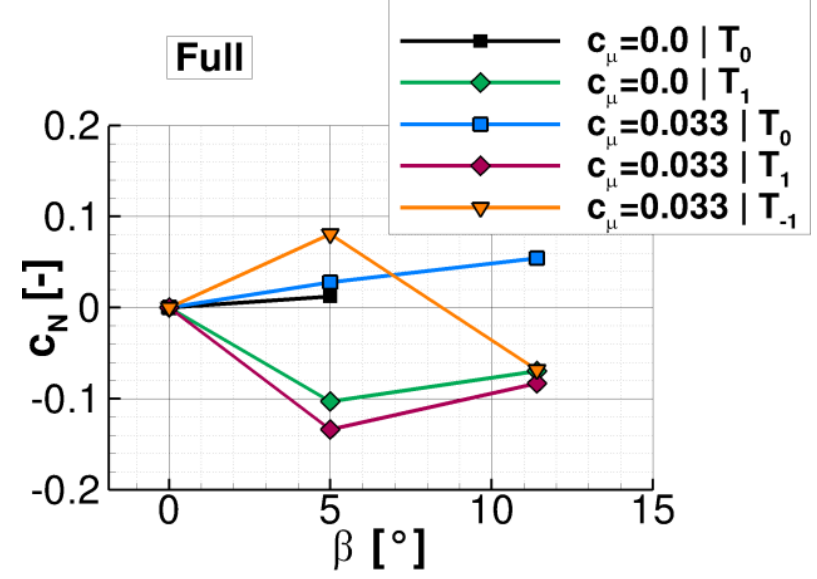

c) Full

Figure 26 Yawing moment dependent on sideslip

\subsection{Lateral Static Behavior}

The lateral static behavior of REFO-2011 was investigated at an angle of attack of $\alpha=0.0^{\circ}$. Besides the zero sideslip case, a moderate sideslip angle of $\beta=5.0^{\circ}$ and a high sideslip angle of $\beta=11.4^{\circ}$ were simulated. The latter corresponds to the maximum required cross flow velocity of $v_{Y}=20$ knots at reference flow conditions.

Fig. 24 plots the pressure distributions in case of zero cross flow for the different circulation control and engine settings at a relative spanwise position of the VTP of $\eta_{V T P}=0.45$. At $\beta=0^{\circ}$, the pressure distributions are symmetric and do not differ in shape. However, with activated circulation control and/or engines, the pressure along the VTP is reduced. At $\beta=5^{\circ}$ (Fig. 25), the main wing wake influenced by circulation control and thrust leads to a reduced suction peak. However, the circulation control also causes a lower pressure in the rear part of the suction side. On the pressure side, the pressure level is reduced in both cases. While the change is rather small for activated circulation control without thrust $\left(c_{\mu}=0.033 \mid \mathrm{T}_{0}\right)$, it is already significant due to thrust only $\left(c_{\mu}=0.0 \mid T_{1}\right)$. It becomes even more visible when both systems are activated $\left(c_{\mu}=0.033 \mid T_{1}\right)$. However, the rotational direction of the propeller plays an important role. If the rotational direction is changed, the pressure is strongly reduced on the suction side and increased at the pressure side.

The trend seen in the pressure distributions can be also identified in the yawing moments of the VTP with respect to sideslip (Fig. 26a). With no thrust, the vertical tail plane reacts with a stabilizing yawing moment to a sideslip angle of $\beta=5^{\circ}$. The difference between activated and deactivated circulation control is negligible, here. When applying thrust, the yawing moment becomes slightly destabilizing, whereas the effect increases with activated circulation control. Changing the rotation of the propeller to inboard down, leads to an increase of the stabilizing yawing moment.

When the sideslip angle is increased to $\beta=11.4^{\circ}$, the impact of the circulation control and thrust appears to be reduced in case of $T_{1}$, resulting in positive yawing moments for all investigated cases. However, if the propeller rotates in inboard down direction $\left(T_{-1}\right)$, the positive yawing moment is reduced, compared to $\beta=5^{\circ}$. The reason for the different sideslip behavior between $T_{1}$ and $T_{-1}$ is based on the different slipstream evolution and its impact on the flow conditions at the rear fuselage. Due to the large flap deflection, the slipstream is

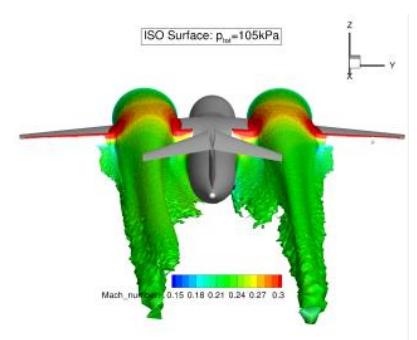

a) $T_{1}$

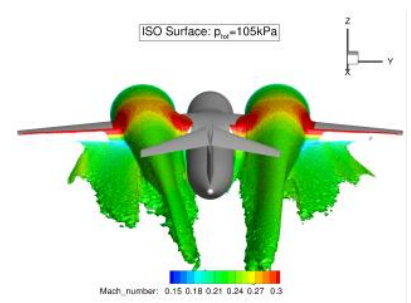

b) $T_{-1}$
Figure 27 Slipstream evolution at $\beta=5^{\circ}$ 
directed downwards behind the wing. The lower part of the slipstream, which is passing below the main wing, is spread towards the sides. Additionally, the cross flow, caused by the sideslip angle, pushes the windward slipstream towards the fuselage (Fig. 27). As a result, under certain conditions, the slipstream is deflected by the fuselage to the lower side. Due to momentum transfer, also the fluid in proximity to the fuselage is accelerated by the slipstream, causing a reduced surface pressure at the lower, windward side of the rear fuselage (Fig. 28). Furthermore, the flow above the fuselage is directed from the leeward side towards the windward side because of mass conservation, leading to a rotating flow around the fuselage.

The sideslip angle, at which this effect appears, depends on the thrust and the rotational direction of the propeller. If the windward propeller rotates in inboard up direction, the slipstream, passing below the main wing, already has a cross flow component towards the fuselage due to the propeller swirl. If the propeller rotates in the opposite direction, the cross flow component of the lower part of the slipstream is pointing away from the fuselage (Fig. 27b). In consequence, the critical sideslip angle is smaller for the case of inboard up rotation (Fig. 28) compared to the inboard down rotation (Fig. 29).

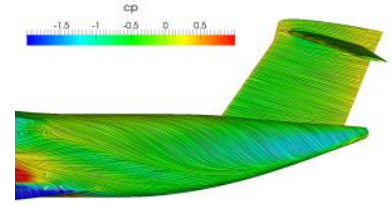

a) $\beta=5^{\circ}$

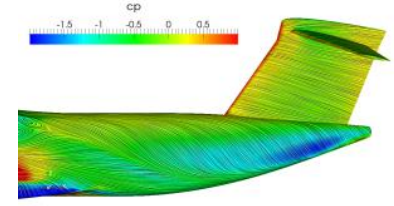

b) $\beta=11.4^{\circ}$
Figure 28 Influence of slipstream on rear fuselage and tail at $T_{1}$ visualized by pressure coefficient distribution and skin friction lines

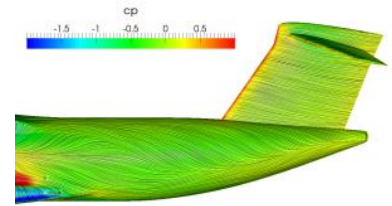

a) $\beta=5^{\circ}$

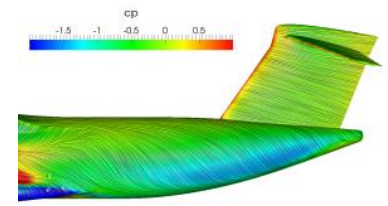

b) $\beta=11.4^{\circ}$
Figure 29 Influence of slipstream on rear fuselage and tail at $T_{-1}$ visualized by pressure coefficient distribution and skin friction lines

Besides the VTP, also the yawing moment of the fuselage is effected by circulation control and thrust (Fig. 26b).

Again, the influence of circulation control is rather small, whereas the influence of thrust is significant. At $\beta=5^{\circ}$, the fuselage also reacts destabilizing on sideslip in case of inboard up rotational direction of the propellers and stabilizing in the opposite case.
However, at $\beta=11.4^{\circ}$, also the thrust with inboard down rotational direction acts destabilizing. As a result, the aircraft is stable in case of deactivated engines and with activated engines and inboard down rotational direction of the propellers for small sideslip angles (Fig. 26c). In case of inboard up direction, the aircraft is laterally unstable for small sideslip angles.

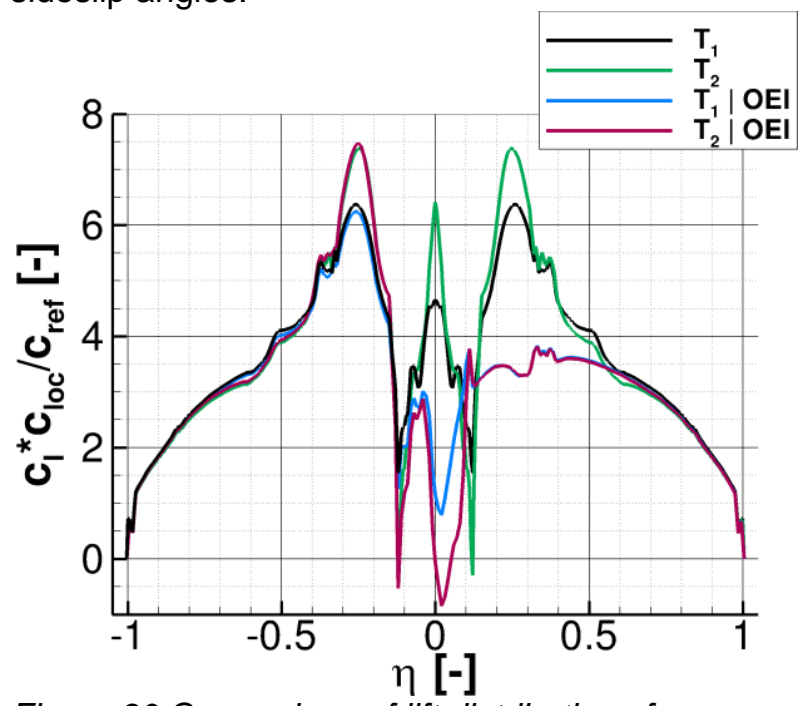

Figure 30 Comparison of lift distributions for symmetric and asymmetric thrust cases

\subsection{System Failures}

For twin-engine aircraft equipped with turboprop engines, the one engine inoperative (OEI) case becomes a dimensioning factor for the VTP sizing. Due to large propellers, the engines have to be mounted further outboard and therefore create significant yawing moments. Furthermore, asymmetric thrust influences the flow around the wing and the tail and thus alters their forces and moments. During take-off and landing, the dynamic pressure and thus the forces of the control devices are low. Therefore, the $O E I$ case has to be particularly considered in high-lift configuration. Since the engines are mounted symmetrically, there is no critical engine regarding OEI. For the simulation of the failure case, the starboard mounted engine is deactivated.

The altered oncoming flow due to the engine deactivation influences the lift distribution of the main wing, as seen in Fig. 30. Without failure case, the lift distribution is fully symmetric and peaks in the upwash region of the propeller. The amplitude of the peak rises with increasing thrust. As expected, with an one-sided system failure, the lift distribution becomes asymmetric. While the distribution on the left wing is only slightly changed, the failure cases lead to severe lift deterioration on the side of the system failure. The 
major lift reduction occurs at the inboard section of the wing in vicinity of the engine.

As a result, the global lift coefficient drops by $12 \%$ at thrust setting $\mathrm{T}_{1}$ and by $18 \%$ at $\mathrm{T}_{2}$ compared to the case with all engines operating (Tab. 2). Also, the pitching moment is influenced. The wing-body pitching moment changes in nose up direction. In contrast, the HTP pitching moment becomes negative due to the reduced downwash at the HTP. As a result, the aircraft tends to pitch up in case of $O E I$.

Table 2 Coefficients of the longitudinal motion in failure cases

\begin{tabular}{|c|c|c|c|c|}
\hline & $\mathrm{cL}$ & $\mathrm{C}_{\mathrm{My}}$ & $\mathrm{C}_{\mathrm{My}, \mathrm{WB}}$ & $\mathrm{C}_{\text {My,HTP }}$ \\
\hline $\mathrm{T}_{1}$ & 3.66 & 0.00 & 0.10 & 0.03 \\
\hline $\mathrm{T}_{2}$ & 3.83 & 0.00 & 0.10 & 0.08 \\
\hline $\mathrm{T}_{1, \mathrm{OEI}}$ & 3.21 & 0.08 & 0.22 & -0.09 \\
\hline $\mathrm{T}_{2, \mathrm{OEI}}$ & 3.15 & 0.19 & 0.42 & -0.13 \\
\hline
\end{tabular}

Due to the asymmetric thrust and lift distribution, the flow behind the wing evolves asymmetrically as well as it is seen in Fig. 31. The slipstream of the operating engine spreads out in spanwise direction and pushes the wake below the fuselage to the starboard side. Above the fuselage, the flow is deflected to the port side, as it was already observed by Mannée [11] and Schroijen [12]. As they also concluded, the asymmetric lift distribution above the wing seems to be the origin of the side wash effect above the fuselage. The fact that a clear pressure gradient can be identified supports this assumption.

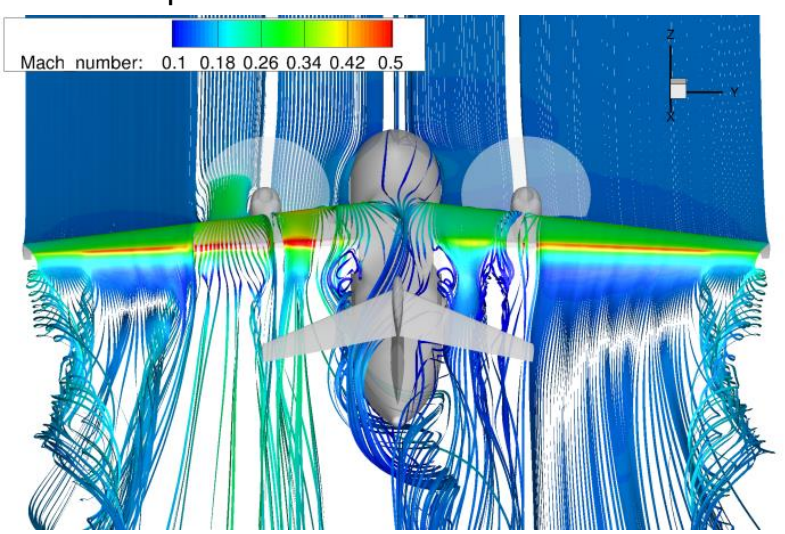

Figure 31 Wake evolution at $T_{1}$ and $O E I$

Tab. 3 gives a detailed overview of the yawing moments for the failure case. As expected, the asymmetric thrust leads to a large negative yawing moment. In case of deactivated circulation control, the yawing moment can be almost completely attributed to the thrust. In contrast, if circulation control is activated, the contribution from the propeller is significantly amplified by additional moments from the fuselage and the VTP, which arise due to the rotational flow around the fuselage. As a result, the yawing moment increases by $\frac{\Delta c_{M Z, A F}}{c_{M z, \text { Prop }}}=126 \%$ at $\mathrm{T}_{1}$ and $\frac{\Delta c_{M Z, A F}}{c_{M z, \text { Prop }}}=$ $88 \%$ at $\mathrm{T}_{2}$. Mostly due to the reduced induced drag of the starboard sided wing, the wing's contribution offsets the aircraft's yawing behavior to some extent.

Table 3 Yawing moments of main contributors and full aircraft in failure case

\begin{tabular}{|c|c|c|c|c|c|}
\hline & \multicolumn{5}{|c|}{$\mathrm{C}_{\mathrm{Mz}}$} \\
\cline { 2 - 6 } & Full & Prop. & VTP & Fuselage & Wing \\
\hline $\begin{array}{c}\mathrm{T}_{1} \mathrm{OEI} \\
\mathrm{C}_{\mu}=0.0\end{array}$ & -0.148 & -0.127 & -0.033 & -0.028 & 0.036 \\
\hline $\begin{array}{c}\mathrm{T}_{1} \mathrm{OEI} \\
\mathrm{C}_{\mu}=0.03\end{array}$ & -0.286 & -0.127 & -0.103 & -0.109 & 0.046 \\
\hline $\begin{array}{c}\mathrm{T}_{2} \mathrm{OEI} \\
\mathrm{C}_{\mu}=0.03\end{array}$ & -0.370 & -0.197 & -0.135 & -0.106 & 0.059 \\
\hline
\end{tabular}

The altered lift distribution due to the engine failure, which was discussed before, also leads to a negative rolling moment (Tab. 4). Additionally, the propeller torque of the running engine adds a rolling moment, which is dependent on its rotational direction. Compared to the wing's share of the rolling moment, the stabilizing contribution from the VTP is rather small.

Table 4 Rolling moments of main contributors and full aircraft in failure cases

\begin{tabular}{|c|c|c|c|c|}
\hline & \multicolumn{4}{|c|}{$\mathrm{C}_{\mathrm{Mx}}$} \\
\cline { 2 - 5 } & Full & Propeller & $\mathrm{VTP}$ & Wing \\
\hline $\begin{array}{c}\mathrm{T}_{1} \mathrm{OEI} \\
\mathrm{C}_{\mu}=0.0\end{array}$ & -0.095 & -0.020 & 0.007 & -0.082 \\
\hline $\begin{array}{c}\mathrm{T}_{1} \mathrm{OEI} \\
\mathrm{C}_{\mu}=0.03\end{array}$ & -0.086 & -0.023 & 0.021 & -0.090 \\
\hline $\begin{array}{c}\mathrm{T}_{2} \mathrm{OEI} \\
\mathrm{C}_{\mu}=0.03\end{array}$ & -0.114 & -0.041 & 0.028 & -0.108 \\
\hline
\end{tabular}

\section{CONCLUSION}

A comprehensive numerical investigation of aerodynamic properties of an aircraft in landing configuration with circulation control and turboprop engines was performed. The analysis of the stall behavior demonstrated a significant influence of wing mounted engine nacelles and thrust regarding the stall mechanism, maximum angle of attack and maximum lift. It is shown that without thrust, the observed degraded stall behavior due to engine nacelles originates from the nacelle vortices, which interact with the main wing boundary layer and circulation control jet. The resulting lift loss can successfully be reduced by the integration of an inboard nacelle strake.

Regarding stability in longitudinal and lateral motion, it is shown that circulation control has a minor effect on the present configuration. Due to 
the use of a T-tail, the horizontal tail plane is far away from the main wing wake. As a result the static and dynamic stability is not significantly impacted by circulation control. In contrast, the static stability is reduced when applying thrust due to an additional positive pitching moment derivative from the propeller and a reduced effectivity of the HTP. The influence of thrust on dynamic stability is to be investigated in the future.

Concerning lateral static stability, it is not negatively influenced by circulation control as such. However, applying thrust can lead to an unstable behavior due to slipstream-fuselage interference effects, which are then amplified by circulation control. The same can be concluded for the case with one engine inoperative. Here, the yawing moment due the engine failure is significantly increased if circulation control is activated.

\section{REFERENCES}

[1] R. J. Englar, G. M. Blaylock and R. J. and Gaeta, "Recent Experimental Development of Circulation Control Airfoils and Pneumatic Powered-Lift Systems," in 48th AIAA Aerospace Sciences Meeting Including the New Horizons Forum and Aerospace Exposition, Orlando, Florida, 2010.

[2] G. K. Korbacher, "Aerodynamics of powered high-lift systems," in Annual Review of Fluid Mechanics, 1974, p. 319-358.

[3] A. Petrov, "Aerodynamics of STOL Airplanes with Powered High-Lift Systems," in Proceedings of the ICAS 2012 Congress, Brisbane, Australia, 2012.

[4] M. Burnazzi and R. Radespiel, "Assessment of leading-edge devices for stall delay on an airfoil with active circulation control," CEAS Aeronautical Journal, vol. 5, no. 4, pp. 359385, 2014.

[5] J. A. Weiberg and C. A. Holzhauser, "STOL Characteristics of a Propeller-Driven, AspectRatio-10, Straight-Wing Airplane with Boundary-Layer Control Flaps, as Estimated from Large-Scale Wind-Tunnel Tests," Ames Research Center, NASA, Moffett Field, California, 1961.

[6] J. Loth, J. Fanucci and S. Roberts, "Flight Performance of a Circulation Controlled STOL Aircraft," Journal of Aircraft, vol. 13, no. 3, pp. 169-173, 1976.

[7] A. J. Pugliese, R. J. Englar and D. W. Taylor, "Flight Testing the Circulation Wing," in AIAA Aircraft Systems and Technology Meeting,
New York, USA, 1979.

[8] J. A. Cochrane, D. W. Riddle, V. C. Stevens and M. D. Shovlin, "Selected Results from the Quiet Short-Haul Research Aircraft Flight Research Program," Journal of Aircraft, vol. 19, no. 2, pp. 1076-1082, 1982.

[9] T. W. Weiss and W. Heinze, "Multidisciplinary Design of CESTOL Aircraft with Powered Lift System," in TU Braunschweig - Campus Forschungsflughafen, Berichte aus der Luftund Raumfahrttechnik, Braunschweig, Shaker Verlag, 2013, pp. 217-230.

[10] D. Keller and R. Rudnik, "Numerical Investigation of Engine Effects on a Transport Aircraft with Circulation Control," Journal of Aircraft, vol. 52, no. 2, pp. 421-438, 2015.

[11] J. Mannée, "Windtunnel Investigation of the Influence of the Aircraft Configuration on the Yawing and Rolling Moments of a TwinEngined Propeller Driven Aircraft with one Engine Inoperative," Amsterdam, 1962.

[12] M. Schroijen and L. \&. S. R. Veldhuis, "Propeller Slipstream Investigation Using the Fokker f27 Wind Tunnel Model with Flaps Deflected," in Proceedings of the ICAS 2008 Congress, Anchorage, 2008.

[13] T. Gerhold, "Overview of the Hybrid RANS Code TAU," Notes on Numerical Fluid Mechanics and Multidisciplinary Design, vol. 89, pp. 81-92, 2002.

[14] P. R. Spalart and S. R. Allmaras, "A OneEquation Turbulence Model for Aerodynamic Flows," in 30th Aerospace Sciences Meeting \& Exhibit, Reno, NV, 1992, 92-0439.

[15] P. R. Spalart and M. Shur, "On the sensitization of turbulence models to rotation and curvature," Aerospace Science and Technology, vol. 1, no. 5, pp. 297-302, July 1997.

[16] A. Raichle, "A new Actuator Disk Model for the TAU Code and application to a sailplane with a folding engine," in Notes on Numerical Fluid Mechanics and Multidisciplinary Design: New Results in Numerical and Experimental Fluid Mechanics VI, Darmstadt, Springer Verlag, 2006, pp. 52-61.

[17] C. O. Marquez Gutierrez, A. Stürmer, C. Clemen and A. Grimminger, "Validation of Actuator Disk Simulations of CROR Propulsion Systems at Low-Speed Flight Conditions," in 30th AIAA Applied Aerodynamics Conference, New Orleans, 2012, 2012-2787. 
[18] C. Lenfers, N. Beck and M. Bauer, "Propeller and Active High LiftWing Interaction in Experiment and Simulation," New Results in Numerical and Experimental Fluid Mechanics $X$, vol. 132, no. Notes on Numerical Fluid Mechanics and Multidisciplinary Design, pp. 51-61, 2016.

[19] A. K. Michler, "Aircraft control surface deflection using RBF-based mesh deformation," International Journal for Numerical Methods in Engineering, vol. 88, pp. 986-1007, 2011.

[20] W. Heinze, C. M. Österheld and P. Horst, "Multidisziplinäres Flugzeugentwurfsverfahren PrADO - Programmentwurf und Anwendung im Rahmen von Flugzeug-Konzeptstudien," in DGLR-Jahrbuch 2001, Bonn, 2001, pp. 17011712.

[21] C. Jensch, K. C. Pfingsten, R. Radespiel, M. Schuermann, M. Haupt and S. Bauss, "DESIGN ASPECTS OF A GAPLESS HIGHLIFT SYSTEM WITH ACTIVE BLOWING," in Proceedings Deutscher Luft- und Raumfahrtkongress, Aachen, 2009.

[22] D. Keller, "Numerical Approach Aspects for the Investigation of the Longitudinal Static Stability of a Transport Aircraft with Circulation Control," New Results in Numerical and Experimental Fluid Mechanics IX, vol. 124, no. Notes on Numerical Fluid Mechanics and Multidisciplinary Design, pp. 13-22, 2014. 